\title{
BUMPER v1.0: a Bayesian user-friendly model for palaeo-environmental reconstruction
}

\author{
Philip B. Holden ${ }^{1}$, H. John B. Birks ${ }^{2,3}$, Stephen J. Brooks ${ }^{4}$, Mark B. Bush ${ }^{5}$, Grace M. Hwang ${ }^{6}$, \\ Frazer Matthews-Bird ${ }^{5}$, Bryan G. Valencia ${ }^{5}$, and Robert van Woesik ${ }^{5}$ \\ ${ }^{1}$ Earth, Environment and Ecosystems, The Open University, Walton Hall, Milton Keynes MK7 6AA, UK \\ ${ }^{2}$ Department of Biology, University of Bergen, P.O. Box 7803, 5020 Bergen, Norway \\ ${ }^{3}$ Environmental Change Research Centre, University College London, London WC1E 6BT, UK \\ ${ }^{4}$ Department of Entomology, Natural History Museum, Cromwell Road, London SW7 5BD, UK \\ ${ }^{5}$ Department of Biological Sciences, Florida Institute of Technology, 150 West University Boulevard, \\ Melbourne, FL 32901, USA \\ ${ }^{6}$ The Johns Hopkins University Applied Physics Laboratory, 11000 Johns Hopkins Road, Laurel, MD 20723, USA \\ Correspondence to: Philip B. Holden (philip.holden@open.ac.uk)
}

Received: 25 August 2016 - Published in Geosci. Model Dev. Discuss.: 21 September 2016

Revised: 11 January 2017 - Accepted: 11 January 2017 - Published: 1 February 2017

\begin{abstract}
We describe the Bayesian user-friendly model for palaeo-environmental reconstruction (BUMPER), a Bayesian transfer function for inferring past climate and other environmental variables from microfossil assemblages. BUMPER is fully self-calibrating, straightforward to apply, and computationally fast, requiring $\sim 2 \mathrm{~s}$ to build a 100taxon model from a 100-site training set on a standard personal computer. We apply the model's probabilistic framework to generate thousands of artificial training sets under ideal assumptions. We then use these to demonstrate the sensitivity of reconstructions to the characteristics of the training set, considering assemblage richness, taxon tolerances, and the number of training sites. We find that a useful guideline for the size of a training set is to provide, on average, at least 10 samples of each taxon. We demonstrate general applicability to real data, considering three different organism types (chironomids, diatoms, pollen) and different reconstructed variables. An identically configured model is used in each application, the only change being the input files that provide the training-set environment and taxon-count data. The performance of BUMPER is shown to be comparable with weighted average partial least squares (WAPLS) in each case. Additional artificial datasets are constructed with similar characteristics to the real data, and these are used to explore the reasons for the differing performances of the different training sets.
\end{abstract}

\section{Introduction}

Transfer functions are numerical tools that are widely used to infer past environment from species (taxon ${ }^{1}$ ) assemblages of microfossils preserved in lake or ocean sediments (Imbrie and Kipp, 1971; Birks and Seppä, 2004; Birks et al., 2010). These taxon assemblages can provide a strong indicator of the environmental characteristics of the habitat in which the microfossils were found. Numerical relationships can be developed between present-day taxon distributions and the environment, and by applying the principle of uniformitarianism (Rymer, 1978), these relationships can estimate palaeo-environments by examining the presence and abundance of palaeo-taxa. Here we take a Bayesian approach and develop a transfer-function model that is tested on three different organism types (chironomids, diatoms, pollen). Although Bayesian transfer functions have a moderately long history (Vasko et al., 2000; Korhola et al., 2002; Haslett et al., 2006) they are still not routinely employed by palaeoecologists, who generally apply frequentist approaches, such as weighted averaging (Birks et al., 1990). The advantages of frequentist approaches are that they are relatively straightforward to understand and apply, and that they are computationally fast. In contrast, Bayesian approaches tend to involve

\footnotetext{
${ }^{1} \mathrm{~A}$ taxon is a biological entity of any taxonomic rank (e.g. family, genus, species or group of morphologically similar species)
} 
more complex mathematics and can be computationally demanding. The advantages of Bayesian approaches, however, are numerous and include the use of prior information (van Woesik, 2013).

The field of Bayesian palaeo-environmental statistics is rapidly developing. Recent work includes the development of a pollen-based multinomial regression model that assumes a Gaussian species response, with joint inference across core time slices (Ilvonen et al., 2016) and a foraminifera-based multinomial non-parametric response model that allows for multi-modal and non-Gaussian taxon response curves (Cahill et al., 2016). Parnell et al. (2016) have published an opensource R package Bclim (Parnell et al., 2015) that uses pollen response surfaces to generate a series of equally probable joint multivariate climate trajectories.

The principal motivation for a Bayesian approach is that the palaeo-environment is treated probabilistically, and can be updated as additional data become available. Bayesian approaches therefore provide a reconstruction-specific quantification of the uncertainty in the data and in the model parameters. Bayesian models are in principle ideally suited to multiproxy reconstructions because the posteriors that are derived from independent proxies can be combined. However, to our knowledge, no Bayesian approach has yet been applied to a multi-proxy reconstruction, at least in part because of the aforementioned difficulties with application, though we note that Cahill et al. (2016) incorporate a second proxy $\left(\delta^{13} \mathrm{C}\right)$ through a prior, assuming a normal likelihood $\sim N\left(\mu_{i}, \tau\right)$ with constant precision $\tau$.

Here we describe the "Bayesian user-friendly model for palaeo-environmental reconstruction" (BUMPER) and demonstrate its general validity and ease of use through applications to both artificial and real datasets. The model can be regarded as a Bayesian analogue to weighted-averagingtype approaches (Birks et al., 1990) because it assumes a unimodal response of each taxon to the reconstructed environmental variable of interest. It therefore shares many of the weaknesses of weighted-averaging-type approaches (Huntley, 2012; Juggins, 2013), most notably by assuming that the responses to different environmental variables are independent. It is the task of the ecologist, assisted by multivariate techniques such as ordination (Hill and Gauch, 1980; Juggins and Birks, 2012; Juggins, 2013), to ascertain which environmental variable is the dominant driver of change in the assemblage through time. When strong interactions are suspected, approaches that concurrently reconstruct the interacting variables may be more appropriate (Huntley, 1993, 2012).

The mathematics of BUMPER was developed and applied to the Surface Water Acidification Programme (SWAP) diatom training set (Stevenson et al., 1991) in Holden et al. (2008). The mathematics is unchanged here. Instead the primary motivation of the present study is to document the developments to the model needed to make it user-friendly. Two important steps were to, first, develop an approach to generalise and automate the priors for the model parameters (we do not assume that the user has the necessary expertise to make these decisions) and, second, demonstrate that the model can be applied to other organism types and environmental variables. We apply the model to both artificial and real data, considering a range of different organism types to demonstrate the model's general applicability.

\section{The model}

The mathematics of BUMPER has been described in detail (Holden et al., 2008). In this section we summarize the underlying philosophy. The principal assumption underlying the model is that a biological taxon exhibits a unimodal response to an environmental variable, so that both the probability of presence and the expected abundance of a given taxon are maximized at the environmental optimum favoured by that taxon. Moreover, the taxon optima are fixed, so that the response to the environmental variable of interest is assumed to be independent of other environmental variables.

\subsection{Parameter estimation}

A species (or taxon) response curve (SRC) defines the probability of an observed count of a species, or taxon, as a function of the environmental variable of interest. An SRC is defined by five (initially unknown) parameters. These parameters specify the environmental optimum and tolerance of each taxon, together with the probability of its presence, and its expected abundance under optimal conditions (see Sect. 2.3 for details).

BUMPER considers 2560 possible SRCs for each taxon ${ }^{2}$ and applies Bayesian parameter estimation to quantify how well each of the SRCs fit the observations of the training set. Bayes equation states that the probability that a model (each of the SRCs) is correct, in light of observational data, is proportional to the probability that the data would be observed if the model were correct. Expressed more formally, for each of the $j=1,2560$ SRCs considered for each taxon $k$, probability weights are derived from the observed environmental variable $x_{i}$ and the taxon count $y_{i k}$ in each training-set site $i$ :

$$
\begin{gathered}
\operatorname{prob}\left(\operatorname{SRC}_{j k} \mid Y_{k}, X\right) \propto \operatorname{prob}\left(\operatorname{SRC}_{j k}\right) \\
\times \prod_{i} \operatorname{prob}\left(y_{i k} \mid \operatorname{SRC}_{j k}, x_{i}\right) .
\end{gathered}
$$

The first term on the right hand side is the prior, the probability that the model is correct before we consider the data. The proportionality constant means that we have to normalise the calculated probabilities. This is achieved from the assumed

\footnotetext{
${ }^{2}$ The model of Holden et al. (2008) considered 8000 SRCs. We here reduce the SRC resolution from $(20,4,5,5,4)$ to $(10,4,4,4$, 4 ) in order to benefit from the $\sim 3$-fold increase in computational speed.
} 
constraint that for each taxon $k$, the following applies:

$\sum_{j} \operatorname{prob}\left(\mathrm{SRC}_{j k} \mid Y_{k}, X\right)=1$.

From now on $\operatorname{prob}\left(\mathrm{SRC}_{j k}\right)$ is the posterior probability, the probability of the SRC given the training data $Y$ and $X$.

\subsection{Environmental reconstruction}

To reconstruct the environment from an observed fossil count $y_{k 0}$ of each taxon $k$ within the fossil assemblage, the probability-weighted SRCs are used to derive likelihood functions for each taxon of the reconstructed variable $x$. Here we again use the Bayes relationship, this time stating that the probability that a reconstructed value is correct in the light of an observed taxon count is proportional to the probability that the taxon count would be observed in that environment. Considering for now a single observed taxon, Bayes' equation can be written as follows:

$\operatorname{prob}\left(x \mid y_{k 0}\right) \propto \operatorname{prob}\left(y_{k 0} \mid x\right) \times \operatorname{prob}(x)$.

We derive a normalized likelihood function for the taxon, considering only the first term on the right hand side of Eq. (3). This allows us to treat the function as a probability distribution of the environmental variable, given no prior knowledge and a count of this single taxon in isolation. As we do not know the true SRC of the taxon with certainty (in general, the calibration will have resulted in many SRCs with non-zero probability), the likelihood function is derived from all significant SRCs, combined using the probability weights calculated in Sect. 2.1, applying the law of total probability:

$$
\begin{gathered}
L_{y}\left(x \mid y_{k 0}\right) \propto \operatorname{prob}\left(y_{k 0} \mid x\right)=\sum_{j} \operatorname{prob}\left(y_{k 0}, \mathrm{SRC}_{j k} \mid x\right) \\
=\sum_{j}\left\{\operatorname{prob}\left(\mathrm{SRC}_{j k}\right) \times \operatorname{prob}\left(y_{k 0} \mid \mathrm{SRC}_{j k}, x\right)\right\} .
\end{gathered}
$$

This expression is evaluated at 100 evenly spaced points across a range that comfortably spans the training-set environmental range (see Sect. 2.4), and is normalized to 1 . (We note that this normalization step is required to build the blended likelihood function in Eq. 6.)

An alternative and less-constrained presence-absence likelihood function can be derived (and normalized as Eq. 4):

$$
\begin{aligned}
& L_{\mathrm{p}}\left(x \mid y_{k 0}\right) \propto \operatorname{prob}\left(y_{k 0}>0 \mid x\right)=\sum_{j}\left\{\operatorname{prob}\left(\mathrm{SRC}_{j k}\right)\right. \\
& \left.\quad \times \operatorname{prob}\left(y_{k 0}>0 \mid \mathrm{SRC}_{j k}, x\right)\right\} .
\end{aligned}
$$

BUMPER derives a linear combination of these two functions, as follows:

$L\left(x \mid y_{k 0}\right)=(1-\eta) L_{y}\left(x \mid y_{k 0}\right)+\eta L_{\mathrm{p}}\left(x \mid y_{k 0}\right)$.

The motivation for this blended likelihood function is that $L_{y}$ provides a tighter constraint on the posterior, but the wide tails contributed by $L_{\mathrm{p}}$ reduce the possibility of the correct solution being ruled out by an outlying taxon, for instance resulting from misidentification or unusual taphonomic processes. We assume $\eta=0.5$, except when building a purely binary, presence-absence model, when $\eta=1.0$. Hereafter, we refer to $L$ simply as the likelihood function of a taxon.

The posterior probability distribution for the reconstructed variable $\operatorname{prob}(x)$ is derived by combining any prior knowledge $\operatorname{prob}\left(x^{\prime}\right)$ with the product of likelihood functions of all considered taxa in the assemblage, as follows:

$\operatorname{prob}(x) \propto \operatorname{prob}\left(x^{\prime}\right) \times \prod_{k} L\left(x \mid y_{k 0}\right)$.

The reconstruction is evaluated at the same 100 points as the likelihood function, and the probabilities are normalized to 1.

A point estimate for the reconstructed variable is derived as follows:

$\hat{x}=\int x \operatorname{prob}(x) \mathrm{d} x$.

A simple uncertainty metric for the reconstruction is derived as follows:

$\Delta=\sqrt{\int(x-\hat{x})^{2} \operatorname{prob}(x) \mathrm{d} x}$.

\subsection{Probability distribution}

To apply Bayes' equation we require an expression for the probability of observing some count $y$ as a function of the environmental variable $x$. The assumption of a unimodal response leads us to assume that the expected abundance (given presence) $N_{i k}$ of taxon $k$ in site $i$ can be fitted by a Gaussian response curve (ter Braak and Barendregt, 1986) about some optimum value $u_{k}$ of the environmental variable $x_{i}$, written as follows:

$N_{i k}=N_{k} e^{-\left(x_{i}-u_{k}\right)^{2} / 2 t_{k}^{2}}$.

Here $N_{k}$ is the expected abundance (given presence) at the taxon optimum, and the tolerance $t_{k}$ is a measure of how rapidly the expected abundance falls off away from this optimum.

The probability of presence (e.g. used to calculate the $L_{\mathrm{p}}$ likelihood function, Eq. 5) is also assumed to follow a Gaussian distribution about the same optimum, though not necessarily of the same tolerance, and can be written in terms of the expected abundance, as follows:

$p_{i k}=p_{k}\left(N_{i k} / N_{k}\right)^{P_{k}}$.

where $P_{k}$ is needed because we do not require the same tolerance for $N_{k}$ and $p_{k}$, although we can achieve this with $P_{k}=1$. We illustrate the role of $P_{k}$ through example; $P_{k} \ll 1$ describes a taxon that is found at high abundance only in an 
environment that is relatively close to its environmental optimum, but may also be present (albeit only at low abundance) far away from this optimum.

Taxon-count distributions are represented with a hurdle model (a zero-inflated distribution with truncation at zero of the distribution of percentage counts). The probability of a non-zero count $y_{i k}$ (used to calculate the $L_{y}$ likelihood function, Eq. 4) is assumed to follow an exponential decay, with the decay constant defined by the expected count $=1 / N_{i k}$, normalized so that the total probability of all (non-zero) percentage counts equals the probability of presence $p_{i k}$, and is expressed as a continuous distribution, truncated at 0 and 100:

$\operatorname{prob}\left(y_{i k} \mid x_{i}\right)=\frac{\left(p_{i k} / N_{i k}\right) \exp \left(-y_{i k} / N_{i k}\right)}{1-\exp \left(-100 / N_{i k}\right)}$.

The denominator normalizes the integral over the range $0<$ $y_{i k} \leq 100$. We note that the denominator is missing from the description of Holden et al. (2008), although it was correctly implemented in the model itself. The probability of absence $\left(y_{i k}=0\right)$ is given by $1-p_{i k}$.

\subsection{Species response curve (SRC) priors}

The SRCs are defined by five parameters: species or taxon optimum $u_{k}$, tolerance $t_{k}$, tolerance scaling $P_{k}$, probability of presence at environment optimum $p_{k}$, and expected abundance (given presence) at environmental optimum $N_{k}$. In general we have little a priori knowledge of appropriate SRC values, and require their priors to be uninformative. However, for computational tractability, we need to eliminate implausible parameter values.

Previous applications (Holden et al., 2008; Matthews-Bird et al., 2016) used uniform priors for the SRC parameters over ranges that were chosen largely on the basis of subjective judgement (and ascribing a probability of zero outside of those ranges). However, requiring subjective judgement is not desirable given our aim to maximize the user-friendliness of the model, and it can be rather time consuming. To prevent subjectivity, BUMPER introduces a simple automated process, based upon an indicative taxon tolerance $t^{\prime}$ that is a characteristic of the training set:

$t^{\prime}=\frac{1}{m} \sum_{k} \sqrt{\frac{1}{n_{k}} \sum_{i}\left(x_{i}-u_{k}^{\mathrm{WA}}\right)^{2}}$.

For each taxon $k$ we consider the environmental variable $x_{i}$ at each site where the taxon is present, and derive a root mean square (RMS) distance from the weighted-average optima, $u_{k}^{\mathrm{WA}}$. We average across the $m$ taxa that have at least two training-set observations $\left(n_{k} \geq 2\right)$. The weighted-average optima are defined in the usual way:

$u_{k}^{\mathrm{WA}}=\sum_{i} y_{i k} x_{i} / \sum_{i} y_{i k}$.
This metric $t^{\prime}$ is used to automate the definition of plausible ranges for taxon optima and tolerance. The approach we take is analogous to precalibrating the parameters of a physical model (Edwards et al., 2011). We only attempt to rule out implausible parameter values and we ascribe equal probabilities to all plausible parameter combinations. The precalibration only seeks to eliminate SRCs with a probability close to zero, and is thereby designed to avoid the trap of over-constraining the posterior by using the training-set data twice.

We consider 10 possible values for the taxon optima, and 4 possible values for each of the 4 other parameters, giving a total of 2560 SRC combinations. The priors for the five SRC parameters are uniform within specified ranges:

1. Optima $u_{k}$ are allowed to take values in the range $\left(x_{\min }-t^{\prime}\right)$ to $\left(x_{\max }+t^{\prime}\right)$, where $x_{\max }$ and $x_{\min }$ are the extremes of the training-set environments. We allow optima beyond the sampled environment range, but optima beyond this extended range are considered unlikely. These ranges are approximately consistent with the subjective priors used in Holden et al. (2008), being training set $\pm 0.5 \mathrm{pH}$ units $\left(t^{\prime}=0.63 \mathrm{pH}\right.$ units), and Matthews-Bird et al. (2016), being training set $\pm 5^{\circ} \mathrm{C}$ $\left(t^{\prime}=3.0^{\circ} \mathrm{C}\right)$.

2. Tolerances $t_{k}$ are allowed to take values in the range $2 t^{\prime} / 3$ to $3 t^{\prime}$. Tolerances at the upper limit are presumably unlikely, and in any event would only weakly constrain the reconstruction. Although tolerances below the lower limit may be reasonable, this choice represents a conservative constraint that, for instance, prevents unrealistically narrow tolerances when a taxon is poorly sampled within a training set. These ranges are consistent with the subjective choices in Holden et al. (2008) of 0.4 to $1.7 \mathrm{pH}$ units, equivalent to $0.63 t^{\prime}$ to $2.7 t^{\prime}$, and Matthews-Bird et al. (2016) of 2 to $10^{\circ} \mathrm{C}$, equivalent to $0.67 t^{\prime}$ to $3.3 t^{\prime}$.

3. The probability of presence at the environmental optimum $p_{k}$ is allowed to take values in the range $p_{k}^{\prime}$ to $2.5 p_{k}^{\prime}$, where $p_{k}^{\prime}$ is the percentage of sites in which the taxon is present. A value below $p_{k}^{\prime}$ is clearly unlikely as the probability of presence at the optimum must be at least as high as the probability of presence across the training set. The upper limit is less straightforward to define; this choice is discussed further below.

4. The expected abundance (given presence) at the environmental optimum $N_{k}$ is allowed to take values in the range $N_{k}^{\prime}$ to $2.5 N_{k}^{\prime}$, where $N_{k}^{\prime}$ is the average count of all non-zero observations across the training set. A value below $N_{k}^{\prime}$ is clearly unlikely as the expected abundance at the optimum must be at least as high as the average abundance across the training set. Again, the choice of upper limit is discussed below. 
5. The tolerance scaling $P_{k}$ (Eq. 11) is allowed to take values in the range 0.2 to 1.0 . Low values $\left(P_{k} \ll 1\right)$ are required to represent taxa that need near-optimum conditions to flourish at high abundance, but can survive even when conditions are far removed from this optimum. Values $P_{k}>1$ can be ruled out because this would (nonsensically) describe a taxon that can flourish at high abundance away from its optimum, but cannot survive there. In Holden et al. (2008), $P_{k}$ was allowed to take values in the range 0.4 to 1.0 . Here we reduce the lower limit to 0.2 , for reasons discussed below.

While aspects of the motivation for ranges 3-5 are discussed above, other aspects are not clearly defined by logical considerations, these being the upper limits for $p_{k}$ and $N_{k}$, and the lower limit for $P_{k}$. These ranges were selected empirically on the basis of application of the model to three training sets, being chironomid-based temperature (MatthewsBird et al., 2016), diatom-based pH (Stevenson et al., 1991) and pollen-based temperature (Bush et al., 2017). We define the posterior value for a parameter to be the probabilityweighted value of that parameter across the 2560 SRCs of the respective taxon. The posterior values of $p_{k}, N_{k}$, and $P_{k}$ for each taxon were sorted from smallest to largest and are plotted sequentially in Fig. 1, for each of the three training sets. The horizontal grid lines represent the four values that each parameter is allowed to take. (Note that although the SRC parameters can only take one of four discrete values, the probability-weighted average can take any value within the prior range). It is apparent that the full range of allowed values is required to describe the responses of all taxa in all three training sets (i.e. because the extremes of the probability-weighted averages approach the extremes of the allowed parameter range). Furthermore it is apparent that a wider range is not required, although there is some suggestion that a slightly higher upper limit for $p_{k}$ could have been allowed for the SWAP data (evidenced by the fact that 7 of the 225 species take precisely the upper limit $p_{k}=2.5 p_{k^{\prime}}$ ).

We note that the indicative tolerance is also used to define the environmental range considered in the reconstruction (Sect. 2.2), from $\left(x_{\min }-6 t^{\prime}\right)$ to $\left(x_{\max }+6 t^{\prime}\right)$. Significant probabilities beyond this range are unlikely given the constraints imposed upon the optima and tolerances. In any event, as with any transfer function, the model should not be applied under suspected extrapolation far beyond the training-set environment.

\section{Artificial training-set data}

The probabilistic framework of BUMPER is well suited to the generation of artificial training sets. In part, our motivation for artificial training sets is to investigate how the characteristics of the assemblage affect the performance of the transfer function. Additionally, an important motivation is to apply BUMPER to a selection of training sets with differ-
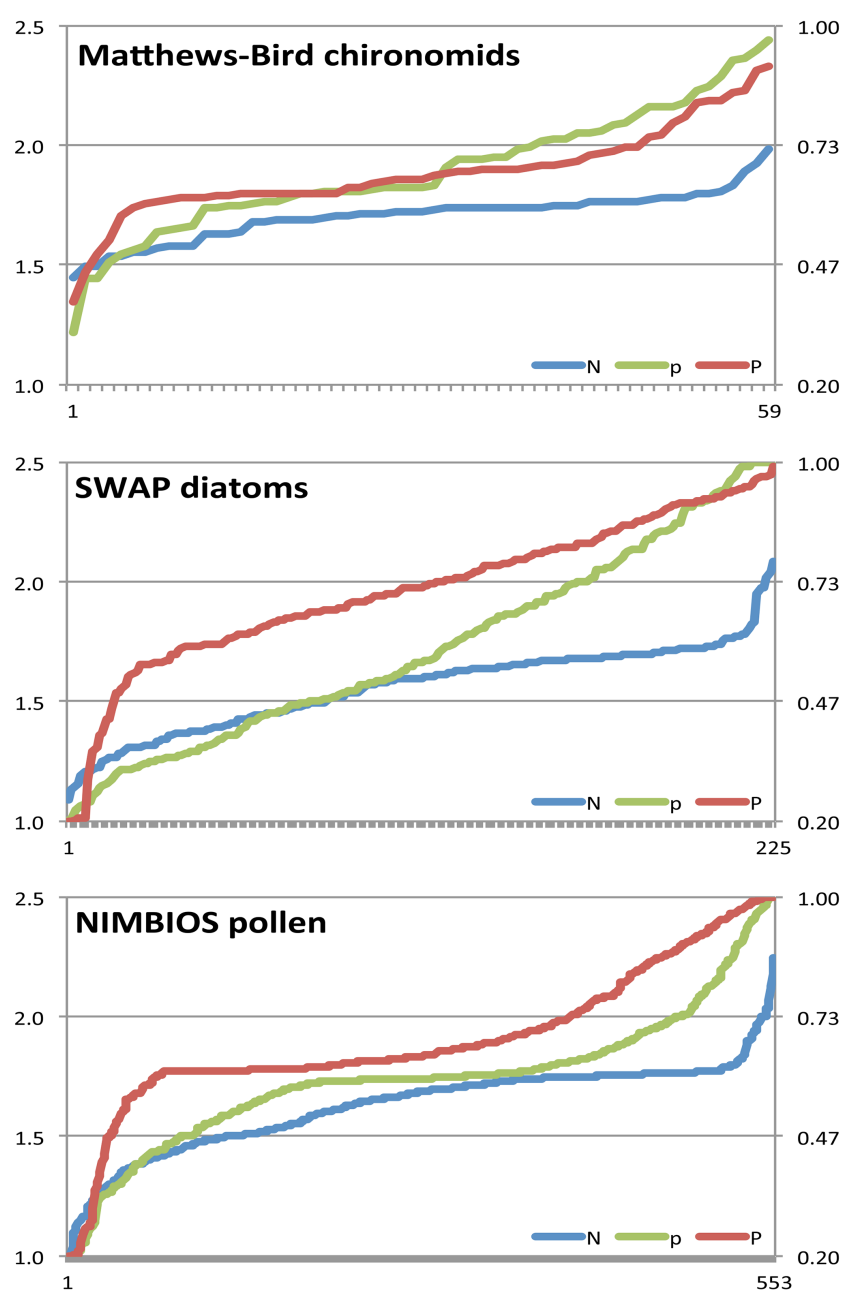

Figure 1. Probability-weighted SRC parameters $N_{k}$ (left-hand axis), $p_{k}$ (left-hand axis) and $P_{k}$ (right-hand axis) are plotted for all taxa. Three training sets are considered: chironomid-based temperature (Matthews-Bird et al., 2016), diatom-based pH (Stevenson et al., 1991), and pollen-based temperature (Bush et al., 2017). The $x$ axes represent the distinct taxa in the training sets (59 chironomid taxa, 225 diatom taxa, and 553 pollen taxa). For each of the three SRC parameters, probability-weighted values are derived for each taxon. These are ordered by increasing value and are plotted sequentially. Horizontal grid lines represent the discrete values allowed within individual SRCs.

ent characteristics to demonstrate that the model can be applied to an arbitrary training set without tuning or user modification. In all of the analyses that follow (in both Sects. 3 and 4) the identical model is applied (although we consider the sensitivity of the model to two important assumptions). This model internally generates precalibrated priors from the characteristic tolerance $t^{\prime}$ and the environmental gradient as described in Sect. 2.4

The artificial data are generated from the BUMPER probabilistic framework. As a result of the generation procedure, the data are idealized and are expected to over-state perfor- 
mance relative to a training set of real data with otherwise similar characteristics. Consider for instance that by applying the probabilistic framework to generate the training set, taxon responses are forcibly defined to be unimodal and with optima that are independent of other environmental variables. Furthermore, there are no misidentifications, nor any possibility of taphonomic error. However, it is worth noting that the artificial training-sites are statistically independent; this eliminates potentially over-optimistic performance statistics that can arise from pseudo-replication in real spatial data that have similar assemblage composition because of geography proximity, and not necessarily because they experience the same environment (Telford and Birks, 2005).

\subsection{Generating artificial training sets}

We generate an artificial training set for an arbitrary environmental variable, under the following assumptions.

i. The training-set sites have an observed environment that is randomly sampled from a uniform distribution over some range $E_{\mathrm{L}}$ to $E_{\mathrm{H}}$. This choice is arbitrary; we select $E_{\mathrm{L}}=100, E_{\mathrm{H}}=200$. The SRC parameters and performance statistics that follow are expressed as a percentage of the environmental gradient $E_{\mathrm{H}}-E_{\mathrm{L}}=100$.

ii. The taxa have optima $u_{k}$ that are randomly sampled from a uniform distribution in the range $u_{\mathrm{L}}$ to $u_{\mathrm{H}}$. Although BUMPER allows optima beyond the sampled gradient, we here simply assume the range of taxon optima is equal to the environmental gradient $\left(u_{\mathrm{L}}=E_{\mathrm{L}}\right.$, $\left.u_{\mathrm{H}}=E_{\mathrm{H}}\right)$.

iii. The taxa have tolerances $t_{k}$ that are randomly sampled from a uniform distribution in the range $t_{\mathrm{L}}$ to $t_{\mathrm{H}}$. The characteristic tolerance of a training set is likely to impact greatly upon the performance of the transfer function because it drives the taxon-turnover rate across the environmental gradient.

iv. The taxa have probabilities-of-presence-at-optimum $p_{k}$ that are randomly sampled from a modified exponential with scale parameter $\beta_{\mathrm{p}}$, defined for $0 \leq p \leq 1$.

$$
f\left(p, \beta_{\mathrm{p}}\right)=\frac{\left(1 / \beta_{\mathrm{p}}\right) \exp \left(-p / \beta_{\mathrm{p}}\right)}{1-\exp \left(-1 / \beta_{\mathrm{p}}\right)}
$$

The denominator adjusts the exponential so that the cumulative probability is 1 at $p=1$. The scale parameter $\beta_{\mathrm{p}}$ is used to tune the assemblage for taxon richness.

v. The taxa's tolerance scaling $P_{k}$ (Eq. 11) are randomly sampled from a uniform distribution in the range 0.2 to 1.0 .

The values of the abundance parameter $N_{k}$ are drawn from a modified exponential distribution (cf. Eq. 15) with scale parameter $\beta_{N}$. The value of $\beta_{N}$ is not an input because an arbitrary choice will lead to an expected total number of counts (i.e. the sum of all taxon counts within a site) that differs from $100 \%$. An appropriate value of $\beta_{N}$ is derived from a Monte Carlo approach; the expected total count is derived from an exploratory 10000 -site training set with $\beta_{N}=10 \%$ and then $\beta_{N}$ is scaled appropriately and the scaled value applied to generate the actual training set. BUMPER does not model a multinomial probability distribution so that percentage counts generated under this approach are not constrained to sum precisely to $100 \%$, even when the appropriate value of $\beta_{N}$ is chosen. A pragmatic approach is taken to address this issue by randomly drawing assemblages, accepting only those with a total count of between 90 and $110 \%$ until the required number of sites has been generated. In accepted sites the counts are scaled to total $100 \%$.

\subsection{Generating transfer functions from the artificial data}

In this section, we cross-validate transfer functions derived from artificial training sets with different characteristics to investigate how these characteristics affect the reconstruction performance. The characteristics we consider are assemblage richness and assemblage tolerance. We define the characteristic assemblage richness as the average number of taxa found in the training-set sites. This is varied in the artificial ensemble through the exponential scale parameter $\beta_{\mathrm{p}}$. A low value of $\beta_{\mathrm{p}}$ ascribes low probabilities of presence, even under optimal conditions, and leads to taxon-poor assemblages. We define the characteristic assemblage tolerance to be the indicative tolerance $t^{\prime}$ (Sect. 2.4). This is adjusted through the prior range for tolerance, $t_{\mathrm{L}}$ to $t_{\mathrm{H}}$. For each assemblage type considered, training sets are generated from 25, 100, 250, and 1000 sites, and are comprised of the same 100 randomly generated artificial taxa.

We consider three assemblage types.

1. Low richness, high tolerance; $\beta_{\mathrm{p}}=10 \%, t_{\mathrm{L}}=15 \%$, $t_{\mathrm{H}}=25 \%$, (solved $\left.\beta_{N}=40 \%\right)$. The assemblage richness that results from these assumptions is 5.7, i.e. the training-set sites contain on average 5.7 taxa. Tolerances are typically $20 \%$ of the environmental gradient.

2. High richness, high tolerance; $\beta_{\mathrm{p}}=50 \%, t_{\mathrm{L}}=15 \%$, $t_{\mathrm{H}}=25 \%$, (solved $\left.\beta_{N}=9 \%\right)$. The prior for tolerance is unchanged from Eq. (1), but the expected probability of presence at the taxon optima is increased, generating taxon-rich assemblages of average richness 18.3.

3. Low richness, low tolerance; $\beta_{\mathrm{p}}=20 \%, t_{\mathrm{L}}=5 \%, t_{\mathrm{H}}=$ $15 \%$, (solved $\beta_{N}=30 \%$ ). The tolerance is halved relative to 1 and the expected probability of presence at the taxon optima is approximately doubled. These two changes together result in a taxon richness of 6.4 , similar to that of Eq. (1), but now the assemblage is comprised of taxa that are more sensitive environmental indicators. 


\subsubsection{WAPLS performance: characterising the assemblages}

Our first objective is to investigate how the characteristics of a training set affect transfer function performance. To achieve this objective we apply the computationally fast weighted average partial least squares (WAPLS) (ter Braak and Juggins, 1993) to large ensembles of training sets. An ensemble approach is necessary because random variability can lead to significantly different transfer function performance, especially for small training sets. For simplicity of interpretation we consider only the first PLS component, equivalent to standard two-way WA regression and calibration with an inverse deshrinking (Birks et al., 1990, 2010). The WAPLS1 ensemble results are plotted as the circles (with associated error bars) in Fig. 2. These points are cross-validated (leave-oneout) RMSEP as a function of the sampling density, defined as the average number of training-set observations per taxon. Each plotted circle in Fig. 2 is derived from 10000 trainingset sites. The sites are randomly generated and assigned to a collection of training sets of the desired size. For instance, to generate an ensemble of 25-site training sets, the 10000 sites are divided into 400 training sets. This enables us to produce robust statistics for each data point, being the mean and standard deviation of the RMSEP, derived across an ensemble of many training sets with similar characteristics.

Unsurprisingly the low richness, high tolerance training sets (assemblage type 1, Sect. 3.2) perform least well. As reconstructions are derived from an average of only six taxa, they are not well constrained and are sensitive to statistical outliers. As the taxa are typically characterized by broad tolerance, they are relatively insensitive environmental indicators. In this assemblage type the cross-validated RMSEP decreases from $16.8 \pm 3.3$ to $10.0 \pm 0.2$ as the training-set size increases from 25 to 1000 sites (sampling density increases from 1.4 to 57). The improvement in performance with training-set size is expected as the WA optima become more accurately defined. However, there is only a modest improvement in performance as the training-set size increases from 250 to 1000 (sampling density increasing from 14 to 57). These diminishing returns are seen across all three of the assemblage types, and together suggest that a requirement for an average of at least 10 observations per taxon is a useful indicator for a well-characterized training set, although we note that idealized data are presumably easier to characterise than real (noisier) data.

An increase in assemblage richness (by a factor of approximately 3 , assemblage type 2 ) or a decrease in assemblage tolerance (by a factor of 2, assemblage type 3 ), both approximately halve the WAPLS1 prediction error (RMSEP reductions of between 37 and $52 \%$ ). We note that while these improvements cannot in general be controlled (they are characteristic of the assemblage), low-richness assemblages are likely to benefit especially from high sample counts (high

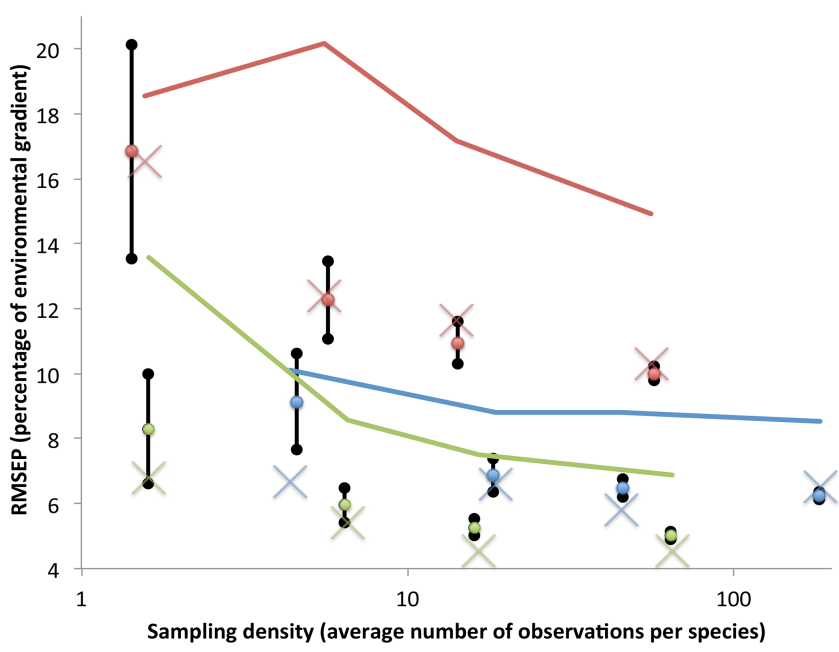

Figure 2. Application to artificial data. Reconstruction performance plotted against the sampling density (the average number of observations per species in the training set). Colour indicates assemblage type: low richness and high tolerance (red), high richness and high tolerance (blue) or low richness and low tolerance (green). Circles with error bars are the mean and standard deviation of the WAPLS1 leave-one-out RMSEP calculated across an ensemble of artificial training sets. From each artificial ensemble, a member is selected that has a WAPLS1 error equal to the ensemble mean. BUMPER is applied to this training set; crosses are the BUMPER leave-one-out RMSEP, solid lines are the BUMPER uncertainty. In summary (see Sect. 3.2), this plot demonstrates the following: (1) reconstruction errors of WAPLS1 (circles) and BUMPER (crosses) are similar for all assemblages. (2) Increasing the sampling density of a training set reduces both the reconstruction errors (circles and crosses) and the BUMPER reconstruction uncertainty (solid lines). However, continued benefits beyond a sampling density of $\sim 10$ are modest. (3) Reconstructions from assemblages that benefit neither from high richness nor from low tolerances (high taxon turnover), plotted in red, are associated with significantly greater error and reconstruction uncertainty. We note that the overstatement of BUMPER uncertainty relative to the reconstruction error (solid lines compared to crosses) is expected for this application to idealized data (see Sect. 3.2.3).

number of counts per training-set site), which tends to increase the observed species richness.

\subsubsection{BUMPER performance and CPU demand}

We now consider the performance of BUMPER. The previous applications of BUMPER (to real ecological data) made two pragmatic assumptions. First, $\eta=0.5$ (Eq. 6), blending the abundance likelihood function $L_{y}$ (Eq. 4) with the presence-absence likelihood function $L_{\mathrm{p}}$ (Eq. 5) so that the wide tails contributed by $L_{\mathrm{p}}$ allow for the possibility of outlying taxon counts. Second, only including taxa that have abundances $>2 \%$ when performing a reconstruction; while very low counts do contribute information, they are generally less informative than high counts, they increase computational load, and they are likely to be less robust. Neither of these 
conservative assumptions is necessary for application to the idealized data considered here. However, we choose to retain them for this analysis in order to avoid the risk of overstating the performance statistics (relative to WAPLS) that are likely to be possible in practice.

The crosses in Fig. 2 plot the leave-one-out RMSEP of BUMPER. For each of the 12 scenarios we select 1 training set, being the first of the randomly generated ensemble members that exhibits a WAPLS1 RMSEP within 0.1 of the ensemble mean, and we use this training set to build a BUMPER model. The performance of this BUMPER model is generally also similar to the ensemble mean WAPLS1 performance. Similar BUMPER and WAPLS performance statistics were previously found with the SWAP diatom-pH training set (Holden et al., 2008) and tropical-Andean chironomids (Matthews-Bird et al., 2016). We note that individual reconstructions (as distinct from the summary statistics of a training set) can differ significantly between the two approaches (Matthews-Bird et al., 2016), in particular because of the increased sensitivity of the reconstruction to "lowcount" taxa (that are only ever observed in low abundance).

The CPU demands of BUMPER are dominated by the calculation of the SRC probabilities (i.e. applying Eq. 1 across all training-set sites for each taxon). This takes $0.022 \mathrm{~s}$ per taxon on a standard personal computer $(2.5 \mathrm{GHz}$ Intel Core i5 processor, 4 GB $1600 \mathrm{MHz}$ DDR3 memory) for a 100-site training set. The CPU demand scales approximately linearly with number of sites. To illustrate, deriving the leave-one-out cross-validated performance of a training set of 500 sites with an average taxon richness of 10 , would require on average $\sim 4.99 \times 10 \times 0.022=1.1 \mathrm{~s}$ per site (i.e. to derive leave-oneout SRC probabilities for 10 taxa from 499 training-set sites), thereby taking $\sim 9$ min to cross-validate the entire training set.

\subsubsection{BUMPER uncertainty}

The solid lines in Fig. 2 plot the BUMPER uncertainty metric $\Delta$ (Eq. 9) from the reconstructions described in Sect. 3.2.2. Although the uncertainties broadly reflect the trends in the reconstruction error, uncertainty is significantly overstated, being $151 \pm 21 \%$ of the RMSEP across the 12 training sets. This overstatement is expected for idealized data because of the blended presence-absence likelihood function $(\eta=0.5$, Eq. 6). This intentionally broadens the likelihood functions to prevent an outlier, such as a taxon misidentification, from ruling out the correct solution. While this assumption has proved useful for real data, it is unnecessarily conservative for idealized data and is therefore expected to overstate uncertainty. Additional BUMPER models (not illustrated) were built for each training set with $\eta=0.0$ and including all taxa present. These models exhibited improved RMSEP relative to the default, reduced by typically $10 \%$. Moreover, these models produced posteriors that better reflect the reconstruction error, with an average uncertainty of $112 \pm 17 \%$
Table 1. Training-set characteristics.

\begin{tabular}{lrrr}
\hline & Chironomids & Diatoms & Pollen \\
\hline Environmental gradient & $25.0^{\circ} \mathrm{C}$ & $2.92 \mathrm{pH}$ units & $24.9^{\circ} \mathrm{C}$ \\
Number of sites & 59 & 167 & 682 \\
Number of taxa & 59 & 225 & 553 \\
Average richness & 7.0 & 50 & 27 \\
Indicative tolerance & $3.2{ }^{\circ} \mathrm{C} / 13 \%$ & $0.63 / 22 \%$ & $3.0^{\circ} \mathrm{C} / 12 \%$ \\
Sampling density & 7.0 & 37 & 33 \\
\hline
\end{tabular}

RMSEP, demonstrating that the uncertainty is meaningfully quantified when the likelihood function is not artificially broadened. (Nevertheless, we retain the broadened likelihood functions for applications to real ecological data.)

\section{Applications to real data}

We consider three training sets of real data, using three different biological proxies and having different assemblage characteristics, summarized in Table 1 . The tropical chironomid (mean annual temperature) dataset of Matthews-Bird et al. (2016) is a 59-site taxon-poor training set comprised largely of taxa with narrow environmental tolerances (expressed as a percentage of the training-set environmental gradient). The SWAP diatom (pH) dataset (Stevenson et al., 1991) is a 167-site taxon-rich training set, significantly larger than the Matthews-Bird dataset, but with broader characteristic tolerances. The NIMBIOS tropical pollen (mean annual temperature) dataset (Bush et al., 2017) is a 682-site species-rich training set that is currently under development and characterized by narrow tolerances. These pollen data are not (in their current state) expected to have the high quality of SWAP, because some taxa are identified to family level whereas other taxa are identified to genus level.

We consider four alternative BUMPER models. First, we test the requirement for a $2 \%$ abundance threshold (Sect. 3.2.2) by generating reconstructions with and without this constraint. Removing this constraint is expected to decrease the uncertainty of the reconstruction (all taxa present are included, thereby narrowing the posterior). We wish to test whether this decreased uncertainty is associated with a reduction in reconstruction error when applied to real data. Second, we consider sensitivity to the form of the likelihood function. The default model applies $\eta=0.5$ (Sect. 2.2). However, in Holden et al. (2008), RMSEP was found to be only weakly dependent upon this parameter so that presenceabsence data alone $(\eta=1.0)$ contain sufficient information to derive a useful predictive model. Compared with the full model, the presence-absence model is expected to increase robustness at the expense of increased uncertainty, with a broader posterior. There may be situations where this conservative approach is preferable, for instance when SRCs are poorly constrained in small training sets, or when misclassification is a concern as the presence-absence model is less 
Table 2. Four BUMPER models. For each of the three training sets (Matthews-Bird et al., 2016; Stevenson et al., 1991; Bush et al., 2017) four models are considered. We use either the standard model $(\eta=0.5)$ or the presence-absence model $(\eta=1.0)$ and reconstruct with either all taxa $\left(y_{k}>0 \%\right)$, or only including taxa with more than $2 \%$ abundance $\left(y_{k}>2 \%\right)$.

\begin{tabular}{lrrr}
\hline (1) $y_{k}>0 \%, \eta=0.5$ & Chironomids & Diatoms & Pollen \\
\hline RMSEP & $2.46^{\circ} \mathrm{C} / 9.8 \%$ & $0.321 \mathrm{pH} / 11.0 \%$ & $2.51{ }^{\circ} \mathrm{C} / 10.1 \%$ \\
Posterior width & $2.74^{\circ} \mathrm{C} / 11.0 \%$ & $0.183 \mathrm{pH} / 6.2 \%$ & $1.64{ }^{\circ} \mathrm{C} / 6.6 \%$ \\
\hline (2) $y_{k}>0 \%, \eta=1.0$ & Chironomids & Diatoms & Pollen \\
\hline RMSEP & $2.40^{\circ} \mathrm{C} / 9.6 \%$ & $0.357 \mathrm{pH} / 12.2 \%$ & $2.73{ }^{\circ} \mathrm{C} / 11.0 \%$ \\
Posterior width & $2.95^{\circ} \mathrm{C} / 11.8 \%$ & $0.204 \mathrm{pH} / 7.0 \%$ & $1.73{ }^{\circ} \mathrm{C} / 6.9 \%$ \\
\hline (3) $y_{k}>2 \%, \eta=0.5$ & Chironomids & Diatoms & Pollen \\
\hline RMSEP & $2.41^{\circ} \mathrm{C} / 9.7 \%$ & $0.369 \mathrm{pH} / 12.6 \%$ & $2.88^{\circ} \mathrm{C} / 11.6 \%$ \\
Posterior width & $3.01{ }^{\circ} \mathrm{C} / 12.0 \%$ & $0.330 \mathrm{pH} / 11.3 \%$ & $2.79^{\circ} \mathrm{C} / 11.2 \%$ \\
\hline (4) $y_{k}>2 \%, \eta=1.0$ & $\mathrm{Chironomids}$ & Diatoms & Pollen \\
\hline RMSEP & $2.27^{\circ} \mathrm{C} / 9.1 \%$ & $0.377 \mathrm{pH} / 12.9 \%$ & $3.04^{\circ} \mathrm{C} / 12.2 \%$ \\
Posterior width & $3.33^{\circ} \mathrm{C} / 13.3 \%$ & $0.473 \mathrm{pH} / 16.1 \%$ & $3.77^{\circ} \mathrm{C} / 15.1 \%$ \\
\hline
\end{tabular}

sensitive to outliers. The cross-validated performances of the four models $\left(y_{k}>2\right.$ or $0 \%, \eta=0.5$ or 1.0$)$ are summarized for each of the three training sets in Table 2, and are plotted for two models $\left(\eta=0.5\right.$ or $\left.1.0, y_{k}>2 \%\right)$ in Fig. 3. Importantly, Fig. 3 illustrates the reconstruction-specific uncertainty $\Delta( \pm 2 \Delta$ is plotted $)$, which in general differs significantly from the training-set RMSEP that is usually assumed to describe the uncertainty of WAPLS approaches.

\subsection{Chironomids (Matthews-Bird et al., 2016)}

The chironomid training set of Matthews-Bird et al. (2016) comprises 59 lakes across tropical South America. The prediction uncertainty associated with this dataset (WAPLS RMSEP $2.4^{\circ} \mathrm{C}$ ) is approximately twice the uncertainty that has been achieved for European chironomid transfer functions, which typically approach $\sim 1^{\circ} \mathrm{C}$ (e.g. Brooks et al., 2012). It has been postulated that this reduced performance is caused by the small size of the training set and uneven sampling across the environmental gradient (Matthews-Bird et al., 2016).

The default model $\left(y_{k}>2 \%, \eta=0.5\right)$ exhibits an RMSEP of $2.41{ }^{\circ} \mathrm{C}$ (Fig. 3a), and $97 \%$ of cross-validated reconstructions lie within $\pm 2 \Delta$ of the observed temperature. However, the best model (RMSEP $2.27^{\circ} \mathrm{C}$ ) is the presence-absence model that requires $2 \%$ abundance to include a species in a reconstruction (Fig. 3b). This model version is the most conservative and might therefore be expected to be the least well performing. However, the training set is small, so the SRCs are not expected to be well defined, and furthermore the number of counts per site is relatively low. These data may therefore favour a more conservative model that does not over-constrain the reconstructions.
When all taxa are included, the reconstruction uncertainty is reduced as expected, from 12.0 to $11.0 \%$ in the default model and from 13.3 to $11.8 \%$ in the presence-absence model. However, this is not accompanied by a reduction in RMSEP. The RMSEP increases slightly from 9.7 to $9.8 \%$ in the default model and from 9.1 to $9.6 \%$ in the presenceabsence model. These data support our choice to retain the $2 \%$ abundance threshold in the default model. Including very low abundance counts increases the computational demand, does not in general improve model performance, and is likely to understate the uncertainty associated with the reconstruction. We note for completeness that the default BUMPER model was also applied in Matthews-Bird et al. (2016) and has a RMSEP of $2.37^{\circ} \mathrm{C}$. The slight difference in performance here results from the different SRC priors used (see Sect. 2.4).

The Matthews-Bird training set can be broadly classified as being low taxon richness (7.0), narrow tolerance (12\%), and relatively poorly sampled (sampling density 7.0). We generated an ensemble of artificial datasets with approximately those characteristics (59 lakes, 59 taxa, $\beta_{\mathrm{p}}=40 \%$, $t_{\mathrm{L}}=8 \%, t_{\mathrm{H}}=18 \%$, yielding an ensemble-averaged species richness of 6.7). The WAPLS1 transfer functions built from these data exhibits a mean RMSEP of $6.3 \pm 0.6 \%$ of the environmental gradient. Although a direct comparison is difficult, given that an improvement in performance is expected under idealized assumptions, these statistics are broadly consistent with those of the real transfer functions (ranging from 9.1 to $9.8 \%$ ).

We consider the training-set characteristics in order to investigate whether they might explain the larger uncertainties of the Matthews-Bird transfer function when compared with European chironomid transfer functions. First, we increase the idealized-analogue training-set size from 59 lakes 


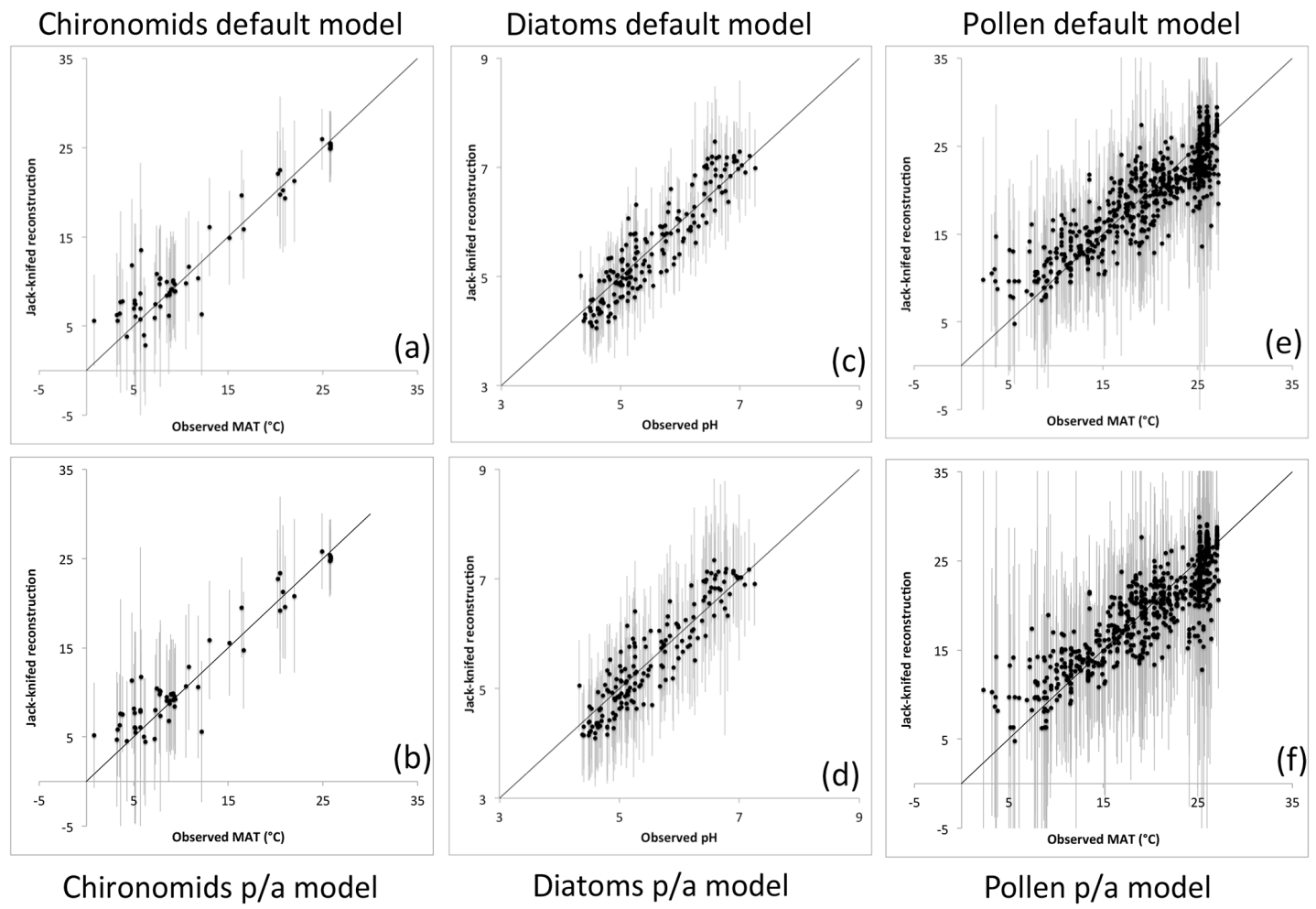

Figure 3. Cross-validated reconstructions (Eq. 8) together with associated uncertainty $\pm 2 \Delta$ (Eq. 9) are plotted against observed training-set environments for each of the three training sets (Matthews-Bird et al., 2016; Stevenson et al., 1991; Bush et al., 2017). We plot the default model $(\eta=0.5)$ and the presence-absence model $(p / a)(\eta=1.0)$. All analyses impose the constraint that $2 \%$ abundance is required for inclusion.

to 118 lakes, thereby better defining the taxon characteristics. This improves the WAPLS1 RMSEP from $6.3 \pm 0.6 \%$ to $5.9 \pm 0.4 \%$. Second, we increase the taxon richness from 6.7 to 13.5 by doubling the number of species to 118 . This improves the WAPLS1 performance to $5.1 \pm 0.5 \%$. Although both factors are likely to contribute to explaining the reduced uncertainty of the European transfer functions, in isolation they do not appear sufficient. We consider a specific example. The Norwegian-chironomid training set of Brooks et al. (2012) comprises 140 taxa sampled from 157 lakes, and was used to construct a WAPLS2 transfer function for July temperature with RMSEP of $1.06^{\circ} \mathrm{C}$ when four outliers are deleted (Brooks et al., 2012). We note that the Matthews-Bird training set reconstructs the mean annual temperature, not the July temperature. The Brooks and Birks training set has an average richness of 24 and an indicative tolerance of $16 \%$. An idealized dataset was built with these characteristics (159 lakes, 140 taxa, $\beta_{\mathrm{p}}=55 \%, t_{\mathrm{L}}=11 \%, t_{\mathrm{H}}=21 \%$, yielding an ensemble-averaged taxon richness of 22) and was found to exhibit a WAPLS1 RMSEP of $5.1 \pm 0.3 \%\left(=0.64^{\circ} \mathrm{C}\right)$. When expressed as a percentage of the environmental gradient, modest improvements in performance are apparent, consistent with that expected from increased training-set size and species richness. However, most of the improvement in pre- dictive power is due to tolerance, but expressed in absolute terms. The Matthews-Bird indicative tolerance is $3.2^{\circ} \mathrm{C}$. The Brooks and Birks indicative tolerance is $2.1^{\circ} \mathrm{C}$. These factors together (increased training-set size, increased taxon richness and, most significantly, narrower absolute tolerances) appear sufficient to explain the improved performances of the Brooks and Birks transfer function over the MatthewsBird transfer function.

\subsection{SWAP (Stevenson et al., 1991)}

The SWAP training set (Stevenson et al., 1991) was developed as part of a substantial scientific effort directed at understanding the impacts of acid rain. Taxonomic workshops resolved problems of nomenclature, splitting and amalgamation of species, and identification criteria (Munro et al., 1990). Approximately 500 counts per sample were made. The training set was statistically pruned (Birks et al., 1990) to leave a high-quality dataset of 267 taxa in 167 sites. The best WAPLS component has RMSEP of $0.310 \mathrm{pH}$ units (Holden et al., 2008).

The best-performing BUMPER model for SWAP is the model that includes all taxa, with no threshold abundance. This model has an $\mathrm{RMSEP}=0.321 \mathrm{pH}$ units. The improvement in performance relative to the default BUMPER 
$\left(y_{k}>2 \%\right)$ threshold (RMSEP $=0.369 \mathrm{pH}$ units, Fig. 3c) may reflect the high quality of the SWAP training set, so that even very low counts are unlikely to be erroneous, and therefore add value to the reconstruction. However, a weakness of the $y_{k}>0 \%$ model is that the posterior widths substantially understate the uncertainty; the low-count taxa narrow the posterior widths by more than is justified, given the modest improvement in performance. Using the default model $\left(y_{k}>2 \%, \eta=0.5\right), 92 \%$ of cross-validated reconstructions lie within $\pm 2 \Delta$ of the measured $\mathrm{pH}$. We note that the performance of the subjectively tuned model in Holden et al. (2008) was $\mathrm{RMSEP}=0.328 \mathrm{pH}$ units .

The SWAP training set can be broadly classified as having high taxon richness (50) and broad tolerance (22\%) and being well sampled (sampling density 37). It is interesting that the RMSEP of this training set is similar to the MatthewsBird set, both exhibiting an RMSEP of approximately $10 \%$ of the environmental gradient. The improvements due to the high quality, taxon richness, and sampling density of SWAP are offset by the loss of precision that is achievable due to broader tolerances.

We generate an artificial training set to mimic the characteristics of SWAP (167 lakes, 225 taxa, $\beta_{\mathrm{p}}=40 \%, t_{\mathrm{L}}=$ $17 \%, t_{\mathrm{H}}=27 \%$, yielding a characteristic taxon richness of 48). The performance statistics of a model derived from these data $5.1 \pm 0.3 \%$ are again broadly consistent with, though significantly better than, the model derived from the real data (11.0 to $12.9 \%)$. The difference between real and artificial data is greater than in the chironomid comparison. Diatoms have been applied as indicators of a wide range of environmental variables including $\mathrm{pH}$, climate, water chemistry, and eutrophication (Smol and Stoermer, 2015), and it may be that the assumption of a single environmental driver of diatomassemblage variability is less well satisfied than it is with chironomids.

\subsection{NIMBIOS (Bush et al., 2017)}

The NIMBIOS dataset (Bush et al., 2017) comprises 682 samples that range from soil samples to mud-water interface samples from lakes. The dataset is of variable quality as it represents a $\sim 30$-year data acquisition effort, in which time there have been substantial improvements in the ability to accurately identify pollen because new pollen keys and descriptions have become available (e.g. Roubik and Moreno, 1991; Bush and Weng, 2007).

The best BUMPER model for NIMBIOS is the full model including all taxa $\left(\eta=0.5, y_{k}>0 \%\right)$, exhibiting an RMSEP $=2.51^{\circ} \mathrm{C}$. This represents a significant improvement to the best WAPLS model $\left(2.92^{\circ} \mathrm{C}\right.$, WAPLS component 2$)$. As with SWAP, however, a weakness of this model is that the posteriors substantially understate the uncertainty. The default model also performs well $\left(\right.$ RMSEP $=2.88^{\circ} \mathrm{C}$ ) and exhibits posteriors that accurately reflect the uncertainty (Fig. 3e), with $93 \%$ of cross-validated reconstructions lying within $\pm 2 \Delta$ of the observed temperature.

The NIMBIOS training set can be classified as having high taxon richness (27) and low tolerance (12\%) and being well sampled (sampling density 33). The training set benefits from optimal characteristics in all three respects. We generate an artificial analogue of NIMBIOS (682 samples, 553 taxa, $\beta_{\mathrm{p}}=15 \%, t_{\mathrm{L}}=7 \%, t_{\mathrm{H}}=17 \%$, yielding a taxon richness of 30). The BUMPER model built from this analogue exhibits an RMSEP of $3.8 \pm 0.1 \%$. This is significantly better than the artificial analogues of the SWAP and Matthews-Bird sets, as would be expected given the optimal characteristics of the training set. However, the performance statistics in this case are substantially better than those of the model built from the real data $(\mathrm{RMSEP}=10.1$ to $12.2 \%)$; the transfer function built from real data does not demonstrate the high performance that might be expected. The discrepancy between the idealized and real data appears to suggest that the quality of the NIMBIOS data is poorer than the other training sets. A major reason for the apparent inaccuracy may be that, unlike the other datasets, pollen is often not recognized to species level ${ }^{3}$ and so a pollen type could contain numerous species that have different environment preferences, and hence may well have a multi-modal distribution rather than the unimodal distribution assumed by BUMPER.

\section{Summary and conclusions}

We have developed BUMPER, a user-friendly implementation of a Bayesian transfer function for palaeo-environmental reconstruction. BUMPER is fully self-tuning, applying a precalibration approach to eliminate implausible values for five model parameters before performing the full probabilistic calibration. The model is straightforward to use, and the only requirement for application to a new training set is to construct the input data files (as described in Appendix A).

We have applied BUMPER to generate transfer functions for chironomid-based temperature (Matthews-Bird et al., 2016), diatom-based pH (Stevenson et al., 1991), and pollen-based temperature (Bush et al., 2017). In each case, cross-validated performance statistics are comparable to the best WAPLS component. The motivation for the model is that it generates a reconstruction-specific uncertainty that, for instance, renders it well suited to multi-proxy reconstructions.

Although the model calibration is performed using all observations, including taxon absences, for reconstruction purposes we only consider taxa found at abundances greater than $2 \%$. When taxa with very low counts are included in a reconstruction, the additional narrowing of the posterior is found to exceed the reduction in reconstruction error (with the Matthews-Bird training set it even leads to slightly increased error), suggesting that the information provided by

\footnotetext{
${ }^{3}$ We note that chironomids are identified to species morphotype level, or genus in some cases.
} 
very low counts is less reliable. We retain the $2 \%$ threshold as we regard the reliable quantification of uncertainty to be more important than the modest improvements in prediction accuracy than can be achieved from including very low taxon counts.

The performance statistics of the three datasets are quite similar, with each displaying a cross-validated error of prediction that is approximately $10 \%$ of the environmental gradient. However, artificial training sets with similar characteristics to the real data revealed that this similarity is largely coincidental and arises for different reasons. The chironomid data are hampered by taxon-poor assemblages, but benefit from narrow-tolerance taxa. Conversely, the diatom data are composed of species with broader tolerances (expressed as percentage of environmental gradient) but the assemblages are substantially richer and taxa are well characterized by the large dataset. The pollen data are large, taxon-rich, and characterized by narrow tolerances. The pollen data were expected to significantly outperform the other training sets; however, this is not found to be the case. The likely explanation is that pollen, unlike the other organism types considered, can be difficult to identify to a low taxonomic (e.g. species) level.

\section{Code availability}

The source code BUMPER1p0.F90, example input data files (Matthews-Bird et al., 2016), and an output visualizer BUMPERpp.R are all provided in the Supplement. Instructions are provided in the Appendices. 


\section{Appendix A: USER MANUAL}

The authors are happy to provide whatever assistance may be needed to run BUMPER. The model is written in FORTRAN 90, included in the Supplement as BUMPER1P0.F90. The open-source GNU Fortran compiler is freely available for MacOS, Linux, and Windows platforms. Once a compiler has been installed, the model is compiled to create an executable BUMPER with the following (linux) command.

\section{\$ gfortran BUMPER1P0.f90 -o BUMPER}

Before running BUMPER, four input data files must be produced and saved in the same directory as the executable file. These files are NAME.lakes, NAME.species, NAME.ts.counts and NAME.core.counts, where "NAME" should be set to a string that identifies the dataset. The Matthews-Bird data have been uploaded to Supplement as an example "MB16".

MB16.lakes: the first line of this file is the number of training-set lakes and is followed by a list of site names together with the environmental variable. All string inputs are limited to 20 characters. Do not include spaces (use an underscore) in character strings. Avoid characters with functionality in R, such as " "”, as these will upset the BUMPERpp plotting software (Appendix B).

MB16.species: the first line is the number of species in the training set, and this is followed by a list of species names.

MB16.ts.counts: a file providing the percentage counts of all species in all training-set sites. The file consists of a row for each lake, each row containing a list of the species counts. It is very important that the rows are ordered identically as they are listed in NAME.lakes and the columns are ordered identically as they are in NAME.species.

MB16.core.counts has the same format as MB16.ts.counts, except the first line is the number of core samples and the first column of the subsequent data is the name of the sample (e.g. depth or age).

The model, can now be run, using the following command.

\section{\$./BUMPER}

The first things that the model will ask for is the name of the training set, in our example MB16. You will then be asked a series of questions:

1. whether to calculate the apparent RMSE (i.e. the fitted model error);

2. whether to calculate the jackknifed (leave-one-out) RMSEP;

3. whether to reconstruct the core;

4. whether to build a presence-absence model $(0==\mathrm{NO}=$ preferred default $)$;
5. whether to apply the $2 \%$ abundance threshold $(1==$ YES $=$ preferred default $)$.

During the input phase, the model will provide the sampling density, mean richness, and indicative tolerance as outputs to the screen. After the input phase, the requested calculations will be performed and various summary outputs will be provided to the screen as the calculations progress. Output files will be created, depending upon the options chosen. These comprise the following:

1. output.data: summary performance statistics, together with individual site reconstructed values and uncertainties;

2. ts_likelihood_app.data: likelihood functions for all species and all training-set sites from the fitted model;

3. ts_likelihood_core.data: jackknifed likelihood functions for all species and all training-set sites (i.e. crossvalidated likelihood functions);

4. core_likelihood.data: likelihood functions for all species and all core samples;

5. reconstruct_ts_app.data: the posteriors of the fitted model;

6. reconstruct_ts_jack.data: the (cross-validated) posteriors of the jack-knifed models;

7. reconstruct_core.data: the core sample posteriors. 


\section{Appendix B: Data visualization with BUMPERpp}

The self-documented tool BUMPERpp. $\mathrm{R}$ is a postprocessing graphical tool written in $\mathrm{R}$, included in the Supplement. The code reads the likelihood data files and generates plots of the reconstructions.

Load the model in $\mathrm{R}$ with the following command.

$$
\text { > source(“BUMPERpp.R”) }
$$

Generate a plot with the following (illustrative) command.

$$
\text { > BUMPERpp(“ts_likelihood_jack.data",1) }
$$

This specifies the input file and sample number to be plotted. Figure B1 provides an illustrative plot.

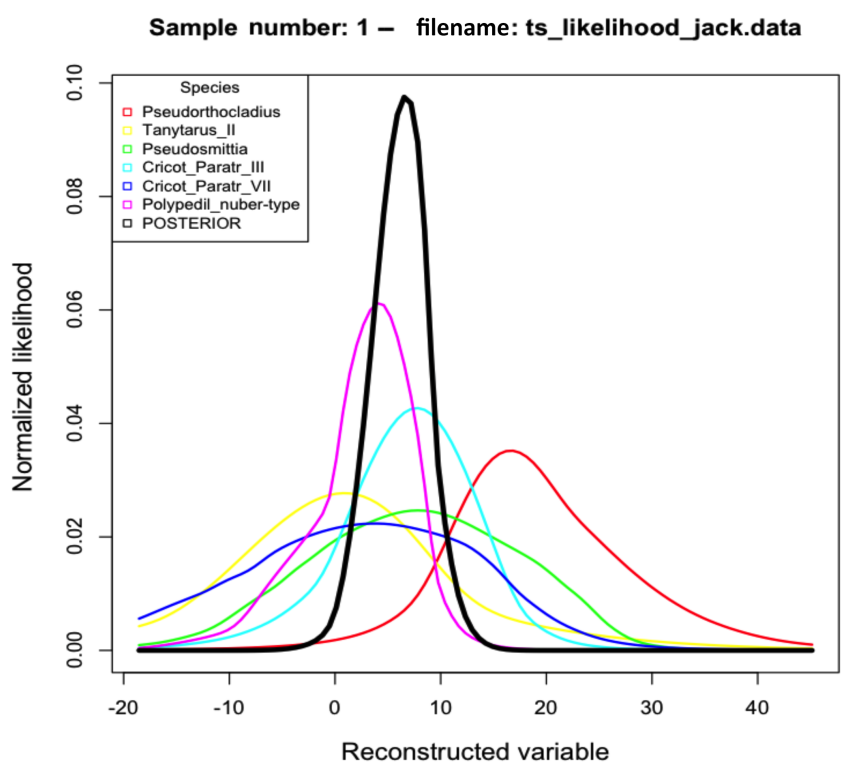

Figure B1. Illustrative output of BUMPERpp.R reconstruction visualization software. 


\section{The Supplement related to this article is available online at doi:10.5194/gmd-10-483-2017-supplement.}

Competing interests. The authors declare that they have no conflict of interest.

Acknowledgements. We are grateful for the careful reviews of Cajo ter Braak and Andrew Parnell, which greatly helped to clarify the mathematical description of the model. Philip B. Holden was funded through the EPSRC project "Research on Changes of Variability and Environmental Risk" (ReCoVER), award RFFER003. Mark B. Bush, Grace M. Hwang, Bryan G. Valencia, and Robert van Woesik were funded through the Climate Proxies Working Group at the National Institute for Mathematical and Biological Synthesis, sponsored by the National Science Foundation through NSF Award no. DBI-1300426, with additional support from the University of Tennessee, Knoxville. Frazer Matthews-Bird was funded by the National Aeronautics and Space Administration (NASA) NNX14AD31G. H. John B. Birks was supported by the Research Council of Norway (projects NoAClim and IGNEX).

Edited by: C. Sierra

Reviewed by: A. Parnell, C. ter Braak, and one anonymous referee

\section{References}

Birks, H. J. B. and Seppä, H.: Pollen-based reconstructions of lateQuaternary climate in Europe - progress, problems and pitfalls, Acta Palaeobot., 44, 317-334, 2004.

Birks, H. J. B., Line, J. M., Juggins, S., Stevenson, A. C., and ter Braak, C. J. F.: Diatoms and pH reconstruction, Philos. T. Roy. Soc. B, 327, 263-278, 1990.

Birks, H. J. B., Heiri, O., Seppä, H., and Bjune, A. E.: Strengths and weaknesses of quantitative climate reconstructions based on lateQuaternary biological proxies, Open Ecol. J., 3, 68-110, 2010.

Brooks, S. J., Matthews, I. P., Birks, H. H., and Birks, H. J. B.: High resolution Lateglacial and early-Holocene summer air temperature records from Scotland inferred from chironomid assemblages, Quaternary Sci. Rev., 41, 67-82, 2012.

Bush, M. B. and Weng, C.: Introducing a new (freeware) tool for palynology, J. Biogeogr., 34, 377-380, 2007.

Bush, M. B., et al.: in preparation, 2017.

Cahill, N., Kemp, A. C., Horton, B. P., and Parnell, A. C.: A Bayesian hierarchical model for reconstructing relative sea level: from raw data to rates of change, Clim. Past, 12, 525-542, doi:10.5194/cp-12-525-2016, 2016.

Edwards, N. R., Cameron, D., and Rougier, J.: Precalibrating an intermediate complexity climate model, Clim. Dynam., 37, 14691482, doi:10.1007/s00382-010-0921-0, 2011.

Haslett, J., Whiley, M., Bhattacharya, S., Salter-Townshend, M., Wilson, S. P., Allen, J. R. M., Huntley, B., and Mitchell, F. J. G.: Bayesian paleoclimate reconstruction, J. Roy. Stat. Soc. A, 169, 395-438, 2006.
Heiri, O. and Lotter, A. F.: Effect of low count sums on quantitative environmental reconstructions: an example using subfossil chironomids, J. Paleolimnol., 26, 343-350, 2001.

Hill, M. O. and Gauch, H. G.: Detrended correspondence analysis: an improved ordination technique, Vegetatio, 42, 47-58, 1980.

Holden P. B., Mackay A. W., and Simpson, G. L.: A Bayesian paleoenvironmental transfer function model for acidified lakes, J. Paleolimnol., 39, 551-566, doi:10.1007/s10933-007-9129-7, 2008.

Huntley, B.: The use of climate response surfaces to reconstruct paleoclimate from quaternary pollen and plan macrofossil data, Philos. T. R.oy Soc. B, 341, 215-223, 1993.

Huntley, B.: Reconstructing palaeoclimates from biological proxies: Some often overlooked sources of uncertainty, Quaternary Sci. Rev., 31, 1-16, 2012.

Ilvonen, L., Holmström, L., Seppä, H., and Veski, S.: A Bayesian multinomial regression model for palaeoclimate reconstruction with time uncertainty, Environometrics, 27, 409-422, doi:10.1002/env.2393, 2016.

Imbrie, J. and Kipp, N. G.: A new micropaleontological method for quantitative paleoclimatology: application to a Late Pleistocene Caribbean core, in: The Late Cenozoic Glacial Ages, edited by: Turekian, K. K., Yale University Press, New Haven, 77-181, 1971.

Juggins, S.: Quantitative reconstructions in palaeolimnology: new paradigm or sick science?, Quaternary Sci. Rev., 64, 20-32, doi:10.1016/j.quascirev.2012.12.014, 2013.

Juggins, S. and Birks, H. J. B.: Quantitative environmental reconstructions from biological data, in: Tracking Environmental Change Using Lake Sediments, Volume 5: Data Handling and Numerical Techniques, edited by: Birks, H. J. B., Lotter, A. F., Juggins, S., and Smol, J. P., Springer, Dordrecht, 431-494, 2012.

Korhola, A., Vasko, K., Toivonen, H. T. T., and Olander, H.: Holocene temperature changes in northern Fennoscandia reconstructed from chironomids using Bayesian modeling, Quaternary Sci. Rev., 21, 1841-1860, 2002

Matthews-Bird, F., Brooks, S. J., Holden, P. B., Montoya, E., and Gosling, W. D.: Inferring late-Holocene climate in the Ecuadorian Andes using a chironomid-based temperature inference model, Clim. Past, 12, 1263-1280, doi:10.5194/cp-12 1263-2016, 2016.

Munro, M. A. R., Kreiser, A. M., Battarbee, R. W., Juggins, S., Stevenson, A. C., Anderson, D. S., Anderson, N. J., Berge, F., Birks, H. J. B., Davis, R. B., Flower, R. J., Fritz, S. C., Haworth, E. Y., Jones, V. J., Kingston, J. C., and Renberg, I.: Diatom quality control and data handling, Philos. T. Roy. Soc. B, 327, 257261, 1990.

Parnell, A. C., Sweeney, J., Doan, T. K., Salter-Townshend, M., Allen, J. R. M., Huntley, B., and Haslett, J.: Bayesian inference for palaeoclimate with time uncertainty and stochastic volatility, J. Roy. Stat. Soc. C-App., 64, 115-138, 2015.

Parnell, A. C., Haslett, J., Sweeney, J., Doan, T. K., Allen, J. R. M., and Huntley, B.: Joint palaeoclimate reconstruction from pollen data via forward models and climate histories, Quaternary Sci Rev., 151, 111-126, doi:10.1016/j.quascirev.2016.09.007, 2016.

Roubik, D. W. and Moreno, P. J. E.: Pollen and spores of Barro Colorado Island, Monographs in Systematic Botany from the Missouri Botanical Garden, St. Louis, Missouri, 1991.

Rymer, L.: The use of uniformitariansim and analogy in palaeoecology, particularly pollen analysis, in: Biology and Quaternary 
environments, edited by: Walker, D. and Guppy, J. C., Australian Academy of Sciences, Canberra, 245-258, 1978.

Smol, J. P. and Stoermer, E. F.: The diatoms: applications for the environmental and Earth sciences, Cambridge University Press, 686 pp., 2015.

Stevenson, A. C., Juggins, S., Birks, H. J. B., Anderson, D. S., Anderson, N. J., Battarbee, R. W., Berge, F., Davis, R. B., Flower, R. J., Haworth, E. Y., Jones, V. J., Kingston, V. J., Kreiser, A. M., Line, J. M., Munro, M. A. R., and Renberg, I.: The Surface Waters Acidification Project Palaeolimnology Programme: modern diatom/lake-water chemistry set, ENSIS, London, 86 pp., 1991.

Telford, R. J. and Birks, H. J. B.: The secret assumption of transfer functions: problems with spatial autocorrelation in evaluating model performance, Quaternary Sci. Rev., 24, 2173-2179, doi:10.1016/j.quascirev.2005.05.001, 2005. ter Braak, C. J. F. and Barendregt, L. G.: Weighted averaging of species indicator values: its efficiency in environmental calibration, Math. Biosci., 78, 57-72, 1986.

ter Braak, C. J. F. and Juggins, S.: Weighted averaging partial least squares regression (WA-PLS): an improved method for reconstructing environmental variables from species assemblages, Hydrobiologia, 269/270, 485-502, 1993.

van Woesik, R.: Quantifying uncertainty and resilience on coral reefs using a Bayesian approach, Environ. Res. Lett., 8, 044051, doi:10.1088/1748-9326/8/4/044051, 2013.

Vasko, K., Toivonen, H. T. T., and Korhola, A.: A Bayesian multinomial response model for organism-based environmental reconstruction, J. Paleolimnol., 24, 243-250, 2000. 